\title{
USO E EFETIVIDADE DE PRÁTICAS DE GESTÃO DA PRODUÇÃO E DO TRABALHO: UM SURVEY DA INDÚSTRIA BRASILEIRA
}

\author{
Adriano de Lemos Alves Peixoto \\ peixoto@hotmail.co.uk \\ Universidade Federal da Bahia - Salvador, BA / Brasil \\ Antônio Virgílio Bittencourt Bastos \\ antoniovirgiliobastos@gmail.com \\ Universidade Federal da Bahia - Salvador, BA / Brasil
}

\begin{abstract}
Recebido em 30/09/2010
Aprovado em 13/10/2011

Disponibilizado em 01/08/2012

Avaliado pelo sistema double blind review

Revista Eletrônica de Administração

Editor: Luís Felipe Nascimento

ISSN 1413-2311 (versão on-line)

Editada pela Escola de Administração da Universidade Federal do Rio Grande do Sul.

Periodicidade: Quadrimestral
\end{abstract}

Sistema requerido: Adobe Acrobat Reader.

\section{RESUMO}

O trabalho buscou caracterizar a adoção, a intensidade do uso, a efetividade e as perspectivas de uso futuro de um conjunto de doze práticas de gestão do trabalho e da produção: Reengenharia de Processos, Terceirização, Parceria na Cadeia de Suprimentos, Cultura de Aprendizagem, Empowerment, Trabalho em Equipe, Manutenção Produtiva Total, Engenharia Simultânea, Tecnologia Integrada por Computador, Célula de Produção, Produção Just-in-time e Gestão da Qualidade Total. Para tanto, foi realizado um survey por meio de entrevistas telefônicas com diretores ou gerentes de produção de 220 empresas industriais com mais de 150 empregados, localizadas em todo o território nacional. Os resultados apontam o uso amplamente difundido do conjunto antes referido de práticas, que foram introduzidas, sobretudo, a partir da última década do século $\mathrm{XX}$ e que são avaliadas pelos gestores de forma bastante positiva, em função dos impactos positivos gerados em termos de qualidade, custos e velocidade de resposta. A pesquisa traça um panorama geral da utilização dessas práticas, fornecendo elementos para estudos intensivos que possam aprofundar a compreensão sobre causas e efeitos dos resultados encontrados.

Palavras-chave: práticas de gestão do trabalho e da produção, adoção de práticas de gestão, organização do trabalho, organização da produção, efetividade de práticas de gestão.

THE USE AND EFFECTIVENESS OF MODERN MANUFACTURING PRACTICES: A SURVEY OF THE BRAZILIAN INDUSTRY 
Uso e efetividade de práticas de gestão da produção e do trabalho: um survey da indústria brasileira

\begin{abstract}
This work seeks to characterize the adoption, intensity of use, effectiveness and the perspective of future use of a group of twelve work and production management practices: Outsourcing; Business Process re-engineering; Supply Chain Management; Learning Culture; Empowerment; Team Based Work; Total Productive Maintenance; Concurrent Engineering; Production Cell; Just in Time; Total Quality Management; Integrated Computer-based Technology. A nationwide sample of 220 companies, with more than 150 employees, of the different industrial sectors was used. Data collection was done by phone interviews with production managers and directors. The findings point toward a broad use of the practices, which have been introduced during the last decade, based on a very good evaluation on its impact over costs, quality and responsiveness. This research also provides a comparative scenario of a nationwide perspective with similar research projects which might deepens the comprehension of the phenomena here described.
\end{abstract}

Keywords: work and production management practices, adoption, intensity of use, effectiveness, perspective of future use.

\title{
USO Y EFICACIA DE LAS PRÁCTICAS DE GESTIÓN DE LA PRODUCCIÓN Y DEL TRABAJO: UN SURVEY DE LA INDUSTRIA BRASILEÑA
}

\section{RESUMEN}

El trabajo buscó caracterizar la adopción, la intensidad de uso, la eficacia y las perspectivas de uso futuro de un conjunto de doce prácticas de gestión del trabajo y la producción: Reingeniería de Procesos, Outsourcing, asociación, cadena de suministro, cultura de aprendizaje, Capacitación, Trabajo en equipo, Mantenimiento Productivo Total, Ingeniería Concurrente, Tecnología Integrada, Informática, Producción de la célula, la producción, JustIn-Time y Gestión de la Calidad Total. Para ello, se realizó un survey por medio de entrevistas telefónicas con los directores o gerentes de producción de 220 empresas industriales con más de 150 empleados en todo el país. Los resultados indican el uso generalizado del conjunto de las prácticas antes mencionadas, que fueron introducidos principalmente de la última década del siglo XX son evaluados por los gerentes sobre la base de los resultados positivos generados en términos de calidad, cuesto y velocidad de respuesta. Esta investigación presenta una visión general del uso de esas prácticas, proporcionando evidencia para estudios intensivos que se puede profundizar en el conocimiento de causas y efectos de los resultados encontrados.

Palabras clave: prácticas de gestión del trabajo y de la producción, adopción de prácticas de gestión, organización del trabajo, organización de la producción, eficácia de lãs prácticas de gestión. 


\section{INTRODUÇÃO}

A literatura gerencial tem sido pródiga na criação e apresentação de práticas inovadoras de gestão da produção e de organização do trabalho como fatores indispensáveis para o sucesso das modernas organizações produtivas, sendo o momento atual caracterizado por uma verdadeira profusão de nomenclaturas e modelos.

Entre as principais transformações em curso, podemos destacar a tendência ao abandono da segurança das linhas de produção fordistas e da gestão taylorista, em direção a formas de organização mais enxutas, baseadas em: delegação de poder e autoridade; trabalhos de equipes; princípios de qualidade aplicáveis não somente a produtos, mas também a processos produtivos (BOXALL; PURCELL, 2003); tecnologias flexíveis e integradas por computador (KARWOWSKI; CHASE, 2005) e mudanças de lay-out das plantas (minifábricas e células, por exemplo) (GITAHY, 2000). Sobre o assunto, Grey (2004) assim se pronuncia:

\footnotetext{
Sem dúvida a TQM ilustra esse ponto. A 'revolução da qualidade' dos anos 1980 tornou-se, nos anos 1990, inadequada em virtude da sua conexão com a 'mudança incremental'. Uma nova revolução se fazia necessária (HAMMER; CHAMPY, 1993), e foi estimulada pela reengenharia. Mas então a reengenharia tornou-se limitada (KOCH; GODDEN, 1996), e novas visões foram oferecidas como soluções. (p.15)
}

Desde o trabalho de Shonberguer (1986) pode-se observar um crescente número de estudos que enfatizam a importância de se adotar práticas e modelos de gestão bem sucedidos como um atalho para o incremento do desempenho das organizações de trabalho e para o aumento da competitividade dos países (BLOOM et al, 2010). A justificativa para tal ação se apóia no argumento de que as empresas líderes nos seus segmentos já identificaram um corpo de conhecimentos, que foi sistematizado em um conjunto de práticas, de modo que os competidores poupariam tempo e recursos ao copiá-las (DAVIES; KOCHAR, 2000) aumentando assim o desempenho das suas organizações. Esse processo mimetismo organizacional tem sido amplamente descrito na literatura gerencial pela Teoria Institucional (DIMAGGIO; POWELL, 1983).

Entretanto, esse mesmo vigor não se observa na investigação mais detalhada sobre o uso e a efetividade dessas práticas. Segundo Caldas (1999), tem-se observado, inclusive, que a introdução e o descarte de novidades gerenciais se dão, muitas vezes, com a mesma intensidade, sem que haja uma adequada avaliação dos seus impactos, fenômeno esse já descrito por ele como de "Difusão de Modismos Gerenciais". 
Uso e efetividade de práticas de gestão da produção e do trabalho: um survey da indústria brasileira

$\mathrm{Na}$ realidade, uma das características da pesquisa no campo dos estudos organizacionais tem sido o uso freqüente do caso único, como podemos perceber, por exemplo, em Araújo e Cabral (2008) e Machado-Silva e Fonseca (2008). Esse padrão de opção metodológica coloca obstáculos sobre a possibilidade de generalizações dos resultados encontrados nesse tipo de estudos. Mesmo quando surveys são realizados, persistem algumas limitações, principalmente porque apresentam alcance geográfico limitado, como em Pelissari, Gonzalez e Vanalli (2011) que investigam pequenas empresas de confecção no Espírito Santo, ou porque se debruçam sobre setores específicos da economia, como em Pacagnella Jr et. al. (2010) que investiga o impacto da inovação no desempenho de empresas de bens de capital no Estado de São Paulo. O resultado é um conjunto de dados desconectados e sem relações evidentes e com poucas possibilidades de generalização para a realidade nacional (MORAES; VASCONCELOS, 2010). Tal situação parece alcançar as mais diversas subáreas dos estudos organizacionais, como se pode constatar em revisões temáticas levadas a cabo nas áreas da gestão de produção (ARKADER, 2003; GODINHO; FERNANDES; LIMA; 2009), Recursos Humanos (TONELLI, et al.; 2003) e Estratégia Empresarial (BERTERO; VASCONCELOS; BINDER, 2003).

Nesse contexto, justifica-se um esforço de pesquisa que busque identificar as práticas mais utilizadas pelas organizações, compreender os motivos que levaram à sua utilização, avaliar os seus impactos e levantar suas perspectivas de uso futuro.

Este trabalho, realizado em um expressivo contingente da indústria nacional, tem como objetivo contribuir para a superação de algumas dessas dificuldades, apresentando um estudo sobre a adoção e a efetividade de um conjunto de doze práticas de gestão do trabalho e da produção desenvolvidos em diferentes setores da economia nacional. Os resultados encontrados permitem estabelecer um marco temporal que serve de referência para processo de difusão, uso e avaliação da efetividade das práticas investigadas.

Além da Introdução, este artigo compõe-se de quatro partes. Na próxima parte, as práticas de gestão pesquisadas serão discutidas a partir de uma taxonomia desenvolvida por Bolden et al. (1997). A vantagem de discutir o processo de adoção dessas práticas a partir de uma taxonomia predefinida reside no fato de que, assim, pode-se ter uma visão de conjunto do comportamento das empresas e dos impactos dessas práticas, além de ser possível realizar comparações entre pesquisas realizadas em diferentes países com base na mesma taxonomia, como, por exemplo, com Waterson et al. (1997), Ide, Takahashi e Kogi (1997), Morrison et. al. (1999), Epitropaki et. al. (2001) e Clegg et. al. (2002). Em seguida, os procedimentos 
metodológicos adotados para a realização da pesquisa de campo são registrados e, na seqüência, os resultados coletados são relatados, enquanto a última parte consignará as análises desses resultados, à guisa de considerações finais.

\section{PRÁTICAS DE GESTÃO DA PRODUÇÃO: DEFININDO O FRAMEWORK DA PESQUISA}

O conjunto de modificações na natureza do processo produtivo tem acarretado uma crescente dificuldade - com a qual se deparam a academia e a indústria - de avaliar adequadamente os impactos de cada nova prática de gestão (BLOOM; VAN REENEN, 2006) antes que uma nova surja (BOLDEN et al., 1997), evidenciando a necessidade de estudos suficientemente abrangentes que produzam dados comparáveis acerca das inúmeras novas práticas gerenciais que vêm sendo adotadas pelas organizações em todo o mundo, desde meados da década de 1970, embora em ritmos diferenciados.

Bolden et al. (1997) apontam ainda para a ausência ou a pouca explicação sobre a escolha das práticas pesquisadas, fazendo com que diferentes estudos utilizem distintos recortes ou níveis analíticos, o que torna ainda mais difícil a comparação dos resultados. Fica, portanto, evidenciada a necessidade de estudos compreensivos que busquem construir um referencial analítico comum para a compreensão do uso e da inter-relação das modernas práticas de gestão do trabalho e da produção. Uma constatação dessa natureza aponta na direção da necessidade de construção de uma taxonomia das práticas. No contexto desse estudo, práticas de gestão são compreendidas e definidas como uma coleção de procedimentos, idéias, valores e ferramentas (LESEURE et al, 2004) que são a expressão de princípios de organização do trabalho e da produção.

Bolden et al. (1997) desenvolveram uma tal taxonomia. Inicialmente, os autores buscaram, por meio de um brainstorm entre pesquisadores ligados à engenharia da produção e ao campo da psicologia organizacional, identificar as práticas de manufatura mais usualmente utilizadas. Nesse estágio, ficou claro que aspectos valorizados por um dos grupos freqüentemente eram omitidos pelos membros do outro, indicando o diálogo reduzido entre distintas disciplinas. A principal conseqüência desse fato pode ser percebida nos freqüentes relatos sobre a dificuldade de implantação de novos procedimentos tecnológicos nas organizações (CAPELLI; NEUMARK, 2001; BRESNAHAN; BRYNJOLFSSON; HITT, 2002), em função da resistência do elemento humano, freqüentemente negligenciado pelo pessoal de operação.

REAd I Porto Alegre - Edição 72 - N 2 - maio/agosto 2012 - p. 372-399 
Uso e efetividade de práticas de gestão da produção e do trabalho: um survey da indústria brasileira

Em uma segunda etapa, os autores buscaram categorizar as principais práticas de gestão da produção. Apoiados em uma extensa revisão de literatura, foram identificadas, inicialmente, 254 práticas de produção e organização que podiam ser consideradas inovadoras sob algum aspecto. Essas práticas foram agrupadas em 87 clusters de práticas semelhantes, posteriormente reclassificados em uma matriz integrada por duas dimensões: ênfase estratégica, que se refere ao foco principal das estratégias diretivas do uso das práticas, e domínio da aplicação, que se refere aos diferentes estágios do ciclo produtivo.

A ênfase estratégica, primeira dimensão criada, reflete simultaneamente preocupações de praticantes e construtos teóricos subjacentes a cada uma das práticas de gestão. Os autores identificaram ainda que tal dimensão abrangia dois focos de análise: o foco no negócio e o foco na organização. O primeiro apontava diretamente para aspectos relacionados à gestão de operações, representando as três prioridades competitivas das áreas de produção: redução de custos, melhoria na qualidade e aumento na velocidade de resposta aos clientes. Por seu turno, o foco na organização representa o núcleo das áreas de engenharia, psicologia organizacional e comportamento organizacional, incluindo um conjunto de práticas mais genéricas, orientadas no sentido do desenvolvimento da organização como um todo, principalmente no que diz respeito à tecnologia e ao desenvolvimento de pessoas.

Essas práticas não têm necessariamente de ser capazes de alcançar, simultaneamente, os três objetivos específicos destinados à produção, ainda que eventualmente possam ser utilizadas para atingir qualquer combinação dos três. Embora as práticas de gestão utilizadas pudessem atingir mais de um objetivo na organização, durante a classificação, de acordo com a ênfase estratégica, Bolden et al. (1997) procuram classificá-las a partir de seu objetivo mais importante. Assim, o Just in Time, por exemplo, foi introduzido com o objetivo primário de redução de custos, ainda que sua utilização permita uma maior flexibilidade na produção e um conseqüente aumento na velocidade de resposta. Dessa mesma forma, a Gestão da Qualidade Total diz respeito à melhoria de qualidade (PRAJOGO, 2005), enquanto a cultura de aprendizagem se relaciona, originariamente, com o desenvolvimento dos empregados (COELHO JÚNIOR; BORGES-ANDRADE, 2008).

Já a segunda dimensão (domínio de aplicação) sobreleva etapas típicas do ciclo produtivo e abarca os componentes-chave do processo de manufatura em si. Dessa forma, as práticas foram agrupadas em quatro categorias, de acordo com a parte da operação na qual ela se encaixava primariamente: design e produção; logística e estoque, que se referem a práticas relacionadas a compras; distribuição ou gestão do estoque; organização do trabalho, que se 
refere especificamente à forma como o trabalho na área de produção é organizado; e uma categoria mais ampla, denominada de organização da manufatura, que cobre práticas e filosofias voltadas para a organização em um sentido mais amplo, a exemplo da prática "produção enxuta".

A partir dessa taxonomia, que organizou o amplo conjunto de práticas inovadoras e de dados que indicavam sua consistência, abrangência e difusão, a pesquisa, conduzida por Waterson et al. (1997), selecionou doze práticas consideradas representativas da variabilidade identificada. O Quadro 1, a seguir, apresenta as doze práticas em suas respectivas células da taxonomia.

\begin{tabular}{|c|c|c|c|c|c|}
\hline \multirow{2}{*}{$\begin{array}{c}\text { Domínio } \\
\text { primário de } \\
\text { aplicação }\end{array}$} & \multicolumn{3}{|c|}{ Foco em Negócios } & \multicolumn{2}{|c|}{ Foco em Organização } \\
\hline & $\begin{array}{c}\text { Melhorias em } \\
\text { qualidade }\end{array}$ & $\begin{array}{l}\text { Redução de } \\
\text { custos }\end{array}$ & $\begin{array}{l}\text { Responsivi- } \\
\text { dade aos } \\
\text { clientes }\end{array}$ & $\begin{array}{r}\text { Tecnologia } \\
\text { melhorada }\end{array}$ & $\begin{array}{c}\text { Desenvolvi- } \\
\text { mento de } \\
\text { funcionários }\end{array}$ \\
\hline $\begin{array}{l}\text { Design e } \\
\text { produção }\end{array}$ & $\begin{array}{l}\text { Manutenção } \\
\text { Produtiva Total } \\
\text { (MPT) }\end{array}$ & $\begin{array}{l}\text { Produção just } \\
\text { in time } \\
\text { (JIT) }\end{array}$ & $\begin{array}{l}\text { Engenharia } \\
\text { Simultânea } \\
\text { (ES) }\end{array}$ & $\begin{array}{l}\text { Tecnologia } \\
\text { Integrada } \\
\text { Baseada em } \\
\text { Computador } \\
\text { (TIC) }\end{array}$ & \\
\hline $\begin{array}{l}\text { Inventário e } \\
\text { estoque }\end{array}$ & \begin{tabular}{|l} 
Parcerias na \\
Cadeia de \\
Suprimento \\
(PCS)
\end{tabular} & $\begin{array}{l}\text { Controle de } \\
\text { Inventário just } \\
\text { in time }\end{array}$ & & & \\
\hline $\begin{array}{l}\text { Organização do } \\
\text { Trabalho }\end{array}$ & & $\begin{array}{l}\text { Terceirização } \\
\text { (TER) }\end{array}$ & $\begin{array}{l}\text { Células de } \\
\text { Produção }(\mathbf{C P})\end{array}$ & & $\begin{array}{l}\text { Trabalho em } \\
\text { Equipes (TEQ) }\end{array}$ \\
\hline $\begin{array}{l}\text { Organização } \\
\text { mais ampla da } \\
\text { produção }\end{array}$ & $\begin{array}{l}\text { Gestão da } \\
\text { Qualidade } \\
\text { Total (GQT) }\end{array}$ & & $\begin{array}{l}\text { Reengenharia } \\
\text { de Processos } \\
\text { (REN) }\end{array}$ & & $\begin{array}{l}\text { Empowerment } \\
\text { (EWP) } \\
\text { Cultura de } \\
\text { Aprendizagem } \\
\text { (CA) }\end{array}$ \\
\hline
\end{tabular}

Quadro 1 - Uma taxonomia de práticas inovadoras em produção e organização

Fonte: BOLDEN et al. (1997).

Embora a busca por flexibilidade seja um objetivo perseguido desde a década de 1950 (MOTTA, 2001), as evidências revelam que tal objetivo tornou-se ainda mais importante nas últimas décadas do século XX e que essa importância acentuar-se-á mais ainda no século XXI (RUBERY, 2006). As práticas de gestão elencadas se reportam à flexibilização que ocorre nas organizações, em suas estruturas, seus processos de gestão e nas relações com fornecedores, 
Uso e efetividade de práticas de gestão da produção e do trabalho: um survey da indústria brasileira

com a finalidade de garantir maior agilidade e capacidade adaptativa frente a demandas contextuais sempre crescentes e desafiadoras.

A introdução desse conjunto de práticas associa-se, por outro lado, ao movimento que diversos autores têm caracterizado como de superação do modelo taylorista-fordista de organização do trabalho e de emergência de uma nova etapa do capitalismo rotulada de pósfordismo (WOOD JR, 2000); ou de especialização flexível (APPELBAUM; BATT, 1994); ou ainda, organização pós-industrial, pós-burocrática, pós-fordista, em rede, federalista, de aprendizado, autoprojetada e colegiada, entre outros (ECCLES; NOHRIA; BERKLEY, 1994). Apesar das discussões teóricas que tal questão suscita, pode-se afirmar que as novas organizações compartilham um conjunto de características básicas, assim descritas por Nonaka e Takeuchi (1997): i) inclinam-se a apresentar uma estrutura mais horizontalizada que a de suas antecessoras; ii) estimulam o empoderamento (do inglês, empowerment) das pessoas; iii) sobrelevam a natureza dinâmica das estruturas organizacionais; iv) enfatizam a importância das competências organizacionais; v) reconhecem o conhecimento como o ativo intangível que mais possibilita a alavancagem de uma organização.

Identificar o nível em que a indústria brasileira adotou práticas de gestão que se inserem naquele movimento global constitui a contribuição central da presente investigação, por gerar dados empíricos que podem melhor balizar as discussões sobre o processo de modernização das empresas nacionais. A pesquisa desenvolvida objetivou responder, prioritariamente, às seguintes questões:

$>$ O quanto as práticas selecionadas são utilizadas?

$>$ Quando as práticas selecionadas foram introduzidas?

$>$ Quais foram as razões principais para a sua introdução?

Quais as práticas vistas como as mais e as menos bem sucedidas?

Quais das práticas terão uso continuado no futuro?

O que pode ser concluído acerca de cada prática?

\section{MÉTODO: TIPO E DESENHO DA PESQUISA}

Este trabalho constitui um recorte de uma pesquisa mais abrangente que buscou caracterizar, no segmento industrial brasileiro, a introdução e o uso das práticas de gestão da produção selecionadas. O recorte efetuado para efeito do presente trabalho e que determinou suas decisões metodológicas guiou-se pelo objetivo central de fornecer uma descrição geral 
da adoção das doze práticas de gestão selecionadas, incluindo os motivos associados à sua adoção, a avaliação do seu êxito e das suas perspectivas futuras.

O estudo se caracteriza como um survey. Assim, com uma característica extensiva e de corte transversal, busca fornecer uma descrição rigorosa de um fenômeno e mostrar como ele se diversifica no âmbito dos diferentes segmentos que caracterizam a população investigada, que então é investigada na busca por padrões de associação (BRYMAN, 2004). Trata-se, também, de um estudo que objetiva alcançar medidas confiáveis da população. Para tanto, o planejamento e a condução de um survey exigem uma atenção especial a três decisões: a construção do instrumento de coleta, de forma a garantir a padronização das informações coletadas; a seleção da amostra, que evitar vieses de representação do universo; e o processo de coleta de informações, que deve minimizar os vieses do entrevistador, para permitir a comparabilidade dos resultados. Essas características gerais sobre a natureza do projeto justificam etapas do processo de investigação realizado:

\subsection{Construção e validação do instrumento de coleta de dados}

Partiu-se de um questionário que funcionou como um roteiro estruturado de entrevista, já disponível, produzido e validado por Waterson et al. (1997). Para a realização do presente estudo, foi feita uma versão do instrumento em português, observando-se os cuidados técnicos para validação desse processo. Nela, alguns itens do questionário original foram eliminados por não se aplicarem à realidade brasileira e outros foram acrescentados em função de interesses específicos do grupo de pesquisa que o aplicou no Brasil.

Foi realizado um teste piloto do questionário para avaliar a qualidade da versão brasileira. O teste foi feito com a utilização de um aparelho de viva voz, o que permitiu o acompanhamento ao vivo das primeiras duas entrevistas, para identificação de possíveis problemas no instrumento e reações dos entrevistados, e possibilitou a criação de um script básico do roteiro de entrevistas.

$\mathrm{O}$ instrumento, integrado por noventa e seis itens fechados, envolve uma matriz com as doze práticas selecionadas - sobre as quais foram feitas várias perguntas específicas que buscam identificar o uso, a efetividade e as razões para a introdução das práticas nas organizações -, estruturadas a partir de três dimensões analíticas básicas: qualidade, nas suas múltiplas dimensões, cabendo ao entrevistado a tarefa de organizar seus referenciais e responder a partir de seus próprios esquemas mentais (BASTOS, 2001), não lhe sendo oferecido um estímulo específico de resposta; custo, de forma similar à qualidade, 
Uso e efetividade de práticas de gestão da produção e do trabalho: um survey da indústria brasileira

considerado como um conceito autoexplicativo; responsividade, ou a velocidade de resposta percebida pelos gestores de produção da organização no atendimento a necessidades específicas dos clientes, definida ainda como velocidade de resposta aos clientes quando os entrevistados apresentaram dúvidas sobre o conceito.

A pesquisa incluiu ainda questões relacionadas: ao ambiente de negócios das organizações (como, por exemplo, o número de plantas industriais, localização, número total de empregados e controle do capital); à prevalência (as razões para implementação) de cada uma das doze práticas investigadas; aos aspectos pertinentes ao desenvolvimento temporal das práticas (o ano de sua introdução, a extensão do uso e as perspectivas de uso futuro); à natureza da força de trabalho (por exemplo, número de trabalhadores temporários, parciais e sindicalizados); aos aspectos vinculados à competição e aos níveis de inovação (como, por exemplo, inovação de produto, e investimentos em pesquisa e desenvolvimento); e aos aspectos atinentes à imprevisibilidade dos mercados, dos fornecedores e dos processos.

\subsection{O desenho da amostra}

A população do estudo foi delimitada como o conjunto de empresas industriais brasileiras, nos seus diversos segmentos, com um número de empregados igual ou superior a 150. O estudo buscou ter uma abrangência nacional, atingindo todos os estados da Federação. Assim, a unidade de análise consistiu de empresas industriais de médio e grande porte que atuavam no Brasil, independentemente de o controle de seu capital ser ou não nacional.

$\mathrm{Na}$ ausência de uma base de dados nacional disponível para delimitar a população, trabalhou-se com várias bases de dados, a saber, os cadastros das Federações Estaduais das Indústrias, a relação das "Maiores e Melhores" da Revista Exame, e ainda o cadastro do Instituto Miguel Calmon (IMiC). Todas essas bases se revelaram incompletas no tocante às informações críticas para a delimitação do universo da pesquisa, sobretudo no que se refere ao quantitativo atualizado de empregados. De posse desse universo delimitado, foi escolhida uma amostra aleatória, estratificada por região e segmento industrial, de 300 empresas, o que asseguraria níveis de confiança aceitáveis para a generalização dos resultados. Ao final do levantamento, foram realizadas 220 entrevistas em todo o território nacional.

As empresas entrevistadas foram organizadas de acordo com o número de funcionários, seguindo-se a classificação adotada pelo Department of Trade and Industry (UK), prevalente na pesquisa original. Esse procedimento visa a manter a comparabilidade dos resultados obtidos na pesquisa nacional com aqueles obtidos nos demais países onde a 
mesma pesquisa foi feita. Assim, na categoria de 150 a 249 funcionários, situaram-se 39,1\% das empresas; na de 250 a 499 funcionários, 34\% das empresas; de 500 a 999 funcionários, 17,7\% das empresas; e com 1000 ou mais funcionários, 9,3\% das empresas. Com relação à participação específica de cada região da Federação na composição da amostra final, verificou-se uma participação superior da região Sul (40,6\%), vindo a seguir a Sudeste $(37,7 \%)$ e a Nordeste, com 19,3\% dos casos. As regiões Norte e Centro-Oeste perfazem apenas 2,4\% dos casos entrevistados. Quanto ao segmento industrial, verificou-se uma participação mais expressiva de empresas dos seguintes ramos: alimentos e bebidas $(12,7 \%)$, metal pesado (11,8\%), química e petróleo $(10,5 \%)$, equipamento eletro-eletrônico $(8,6 \%)$, móveis e madeira $(8,2 \%)$, pedra, vidro e concreto $(8,2 \%)$, papel $(7,7 \%)$, maquinaria industrial e comercial $(7,7 \%)$.

\subsection{O processo de coleta de dados}

Seguindo o padrão da pesquisa original desenvolvida na Inglaterra, o informante entrevistado foi o gestor (diretores, gerentes e supervisores) encarregado da área de operações e produção. Os dados foram coletados por meio de entrevistas telefônicas. Um Call Center foi contratado para a realização das entrevistas. Os operadores foram treinados, e um script foi desenvolvido para a aplicação dos questionários. Um grupo de alunos de iniciação científica, mestrado e doutorado acompanhou a aplicação das entrevistas como procedimento de auditoria e recurso auxiliar em caso de dúvidas.

$\mathrm{Na}$ realização da coleta de dados, foi apresentada aos entrevistados uma breve descrição de cada prática a ser investigada. Em seguida, as questões foram formuladas e, na seqüência, eram apresentadas as respostas possíveis. Em algumas partes do questionário, um possível silêncio do respondente ou uma negativa de escolha de uma das alternativas disponibilizadas foram considerados como respostas válidas e assinaladas pelos entrevistadores. As entrevistas tiveram uma duração média de uma hora tendo sido realizadas no segundo semestre de 2002.

\subsection{O processo de análise de dados}

Os dados coletados são de natureza quantitativa, sendo as categorias previamente definidas no próprio instrumento, e foram digitados e analisados através do SPSS (Statistical Package for Social Sciences). Para fornecer esse panorama descritivo geral do país, foram utilizadas rotinas específicas do SPSS que forneciam estatísticas descritivas - frequiências 
Uso e efetividade de práticas de gestão da produção e do trabalho: um survey da indústria brasileira

percentuais e escores médios - das diversas dimensões ou aspectos utilizados para caracterizar cada prática de gestão.

\section{RESULTADOS}

Os resultados da presente pesquisa possuem, claramente, um caráter descritivo, como já se pode perceber a partir das questões e do tipo de pesquisa realizada. $\mathrm{O}$ interesse, portanto, consiste em oferecer um amplo painel de como as diferentes práticas de inovação foram implementadas e avaliadas no Brasil. Para organizar os dados, este segmento estrutura-se a partir das questões que orientaram a investigação.

\subsection{A intensidade do uso: o quanto essas práticas são utilizadas?}

A primeira e mais básica questão envolvida no levantamento consistiu em verificar a extensão de uso das práticas de inovação. Os resultados obtidos encontram-se na Tabela 01, que especifica os valores médios da intensidade do uso atual (no momento da pesquisa) de cada uma das práticas apresentadas, agrupadas por região geográfica. Os valores apresentados podem variar de 0 (zero=nada), indicando que a prática não está sendo utilizada, a 4 (quatro= totalmente) indicando que a prática está sendo utilizada fortemente pelas organizações investigadas. Os valores intermediários, 1, 2 e 3 (um , dois e três), indicam que a prática está sendo utilizada pouco, moderadamente ou muito, respectivamente.

Tabela 01. Intensidade de uso das práticas por região.

\begin{tabular}{|c|c|c|c|c|c|}
\hline \multirow[b]{2}{*}{ Prática } & \multicolumn{5}{|c|}{ Intensidade de uso por região } \\
\hline & $\begin{array}{l}\text { Sudeste } \\
(\mathrm{N}=81)\end{array}$ & $\begin{array}{c}\text { Sul } \\
(\mathrm{N}=87)\end{array}$ & $\begin{array}{c}\text { Nordeste } \\
(\mathrm{N}=\mathbf{4 1})\end{array}$ & $\begin{array}{l}\text { Norte } \\
(\mathrm{N}=6)\end{array}$ & $\begin{array}{c}\text { Centro-Oeste } \\
(\mathrm{N}=5)\end{array}$ \\
\hline \multicolumn{6}{|c|}{ Design e produção/ Inventário e estoque } \\
\hline TIC & 3,09 & 3,45 & 2,78 & 2,8 & 3,2 \\
\hline $\mathbf{E S}$ & 2,5 & 2,66 & 2,12 & 2,5 & 1 \\
\hline JIT & 2,95 & 3,0 & 2,88 & 3,0 & 2,4 \\
\hline MPT & 2,66 & 2,68 & 2,35 & 3,2 & 2,8 \\
\hline PCS & 3,32 & 3,46 & 3,33 & 3,2 & 4,0 \\
\hline \multicolumn{6}{|c|}{ Organização do trabalho } \\
\hline TEQ & 3,62 & 3,63 & 3,58 & 4,0 & 4,6 \\
\hline $\mathbf{C P}$ & 2,66 & 2,85 & 2,29 & 3,0 & 2,4 \\
\hline TER & 2,33 & 2,38 & 2,23 & 1,8 & 2,6 \\
\hline
\end{tabular}


Adriano de Lemos Alves Peixoto \& Antônio Virgílio Bittencourt Bastos

\begin{tabular}{|c|c|c|c|c|c|}
\hline EWP & 3,08 & 3,08 & 3,13 & 3,6 & 3,2 \\
\hline CA & 3,31 & 3,29 & 3,2 & 3,8 & 3,2 \\
\hline REN & 2,51 & 3,15 & 2,63 & 3,2 & 3,4 \\
\hline GQT & 3,74 & 3,8 & 3,46 & 3,5 & 3,2 \\
\hline $\begin{array}{c}\text { Média por } \\
\text { Região }\end{array}$ & $\mathbf{2 , 9 8}$ & $\mathbf{3 , 1 1}$ & $\mathbf{2 , 8 3}$ & $\mathbf{3 , 1 3}$ & $\mathbf{3 , 0 0}$ \\
\hline
\end{tabular}

Fonte: autores

É importante observar que, para as regiões Norte e Centro-Oeste, o tamanho (N) da amostra é pequeno, o que certamente introduz alguma distorção nos resultados e prejudica a exata avaliação das diferenças entre as regiões, ainda que suas médias gerais se situem dentro do padrão geral encontrado para o restante do país.

Quando as práticas são comparadas uma a uma, por região, percebem-se pequenas diferenças na intensidade de uso atual de cada prática, que, de um modo geral, não são estatisticamente significativas. ${ }^{1} \mathrm{O}$ EWP tem uso mais intenso na região Nordeste, com média 3,13, ao passo que, no Sul e no Sudeste, ele se apresenta com valor de uso atual de 3,08. Já a GQT é de uso mais intenso na região Sul do país $(3,8)$, situando-se em seguida o Sudeste $(3,74)$ e o Nordeste $(3,46)$. Já a CA tem seu uso mais intenso na região Sudeste do país.

Entretanto, quando observamos os resultados a partir de uma perspectiva mais ampla que não a das práticas individualizadas, mas do seu conjunto -, percebemos uma diferença entre as regiões, o que espelha, em alguma medida, as diferenças econômicas existentes em relação ao Nordeste, que apresenta os valores de uso mais baixos para as práticas investigadas. Porém, mais do que diferenças econômicas, os resultados podem ser entendidos como indicadores de dinâmica organizacional, ou seja, eles espelham transformações em curso em um determinado espaço geográfico. Na maioria das vezes, esse espaço é simultaneamente físico e econômico, mas essa relação não parece ser necessária, já que indicadores elevados de uso podem refletir um processo de modernização em uma região atrasada, onde novos processos e técnicas estão sendo rapidamente introduzidos. Comparativamente, uma região bastante dinâmica pode apresentar índices muito semelhantes de uso das práticas, sem que nela esteja sendo introduzido algum elemento novo.

Na Tabela 02, podemos visualizar a intensidade de uso das práticas para os três setores de maior participação nos resultados encontrados da pesquisa: alimentos e bebidas, química e petróleo, metal pesado.

\footnotetext{
${ }^{1}$ Para a avaliação dessas diferenças, realizamos uma análise de variância (ANOVA) entre as regiões Sul, Sudeste e Nordeste. Optamos por excluir as regiões Norte e Centro-Oeste desse procedimento, em função do reduzido número de empresas na amostra, o que tem o potencial de enviesar os resultados.
}

REAd I Porto Alegre - Edição 72 - Nº 2 - maio/agosto 2012 - p. 372-399 
Uso e efetividade de práticas de gestão da produção e do trabalho: um survey da indústria brasileira

Ainda que algumas diferenças possam ser percebidas na comparação dos resultados, geralmente os setores apresentam um padrão de uso médio (média=3). A pequena diferença, a menor, observada para o setor de A\&B não diverge fundamentalmente desse padrão.

Tabela 02: Intensidade de uso em três setores da indústria nacional.

\begin{tabular}{|c|c|c|c|c|}
\hline \multirow[b]{2}{*}{ Prática } & \multirow[b]{2}{*}{$\begin{array}{l}\text { Resultado geral } \\
\qquad(\mathrm{N}=\mathbf{2 2 0})\end{array}$} & \multicolumn{3}{|c|}{ Intensidade de uso por setor } \\
\hline & & $\begin{array}{c}\text { Alimentos e } \\
\text { bebidas }(\mathrm{N}=28)\end{array}$ & $\begin{array}{l}\text { Química e petróleo } \\
\qquad(\mathrm{N}=23)\end{array}$ & $\begin{array}{l}\text { Metal pesado } \\
(\mathrm{N}=26)\end{array}$ \\
\hline \multicolumn{5}{|c|}{ Design e produção/ Inventário e estoque } \\
\hline TIC & 3,18 & 2,75 & 3,26 & 3,19 \\
\hline ES & 2,49 & 2,07 & 2,13 & 2,71 \\
\hline JIT & 2,94 & 2,7 & 2,96 & 2,81 \\
\hline MPT & 2,63 & 2,86 & 2,35 & 2,36 \\
\hline PCS & 3,38 & 3,46 & 3,04 & 3,31 \\
\hline \multicolumn{5}{|c|}{ Organização do trabalho } \\
\hline TEQ & 3,67 & 3,46 & 3,65 & 3,77 \\
\hline $\mathbf{C P}$ & 2,67 & 2,46 & 2,38 & 2,92 \\
\hline TER & 2,3 & 1,96 & 2,87 & 2,31 \\
\hline \multicolumn{5}{|c|}{ Organização mais ampla da produção } \\
\hline EWP & 3,12 & 3,18 & 2,96 & 3,15 \\
\hline $\mathbf{C A}$ & 3,3 & 3,11 & 3,43 & 3,46 \\
\hline REN & 2,84 & 2,86 & 3,35 & 2,84 \\
\hline GQT & 3,71 & 3,22 & 4,0 & 3,92 \\
\hline Média & 3,01 & 2,84 & 3,03 & 3,06 \\
\hline
\end{tabular}

Fonte: autores

De um modo geral, os resultados apresentados para a amostra, quando separada por setor, apresentam o mesmo padrão geral daquele para a população, sendo as diferenças não significativas estatisticamente. Por outro lado, tanto o setor de química e petróleo quanto o setor de metal pesado são tradicionalmente muito mais internacionalizados e intensivos de capital do que o setor de alimentos e bebidas. Assim, os resultados parecem apontar na direção de um movimento de adoção generalizada das práticas pela indústria nacional, independentemente de região, setor ou tecnologia de processo, o que nos leva a imaginar a causa de um movimento de tal intensidade e amplitude.

\subsection{A história: quando as práticas selecionadas foram introduzidas?}


Nas empresas onde uma determinada prática encontrava-se em uso, os entrevistados foram requisitados a identificar o ano de sua introdução. Os resultados apresentados na Tabela 03 estão sumarizados por décadas, sendo criadas seis categorias: antes de 1960, entre 1960 e 1969, entre 1970 e 1979, entre 1980 e 1989, entre 1990 e 1997 e no período de 1998 a 2002. Essa distribuição temporal segue o padrão da pesquisa desenvolvida em Sheffield, o que permite manter os mesmos parâmetros conceituais.

Algumas diferenças merecem ser destacadas: as práticas mais recentemente introduzidas no Brasil são MPT (94,6\% na década de noventa e 56,3\% a partir de 1997) e EWP (93,7\%, sendo que 56,0\% dos casos ocorrem após 1997). Com elevados percentuais de introdução na década de 90, encontram-se: REN (92,7\%), TIC (92,6\%) e JIT (92,0\%). As práticas mais utilizadas entre os anos de 1980 e 1989 foram ES (10,1\%), TEQ (9,7\%), CP $(9,4 \%)$ e CA $(9,1 \%)$. É interessante observar que, para quase todas as práticas, existem referências, ainda que residuais, aos períodos da década de oitenta e anteriores, o que nos mostra claramente a existência e o uso das práticas algum tempo antes de sua generalização.

Tabela 03: Intensidade de uso e ano de introdução

\begin{tabular}{|c|c|c|c|c|c|c|}
\hline \multirow{2}{*}{$\begin{array}{c}\text { Intensidade de } \\
\text { uso }\end{array}$} & \multirow[b]{2}{*}{ Prática } & \multirow{2}{*}{$\begin{array}{c}\text { Intensidade } \\
\text { de uso }\end{array}$} & \multicolumn{4}{|c|}{ Período de introdução } \\
\hline & & & $\begin{array}{c}\text { Antes de } \\
1980\end{array}$ & $\begin{array}{c}\text { Entre 1980- } \\
1989\end{array}$ & $\begin{array}{c}\text { Entre 1990- } \\
1997\end{array}$ & Após 1997 \\
\hline \multirow{2}{*}{$\begin{array}{l}\text { Bastante } \\
\text { Intenso }\end{array}$} & TEQ & 3,67 & 2,6 & 9,7 & 42,3 & 45,4 \\
\hline & GQT & 3,71 & 0 & 8,5 & 50,3 & 41,2 \\
\hline \multirow{4}{*}{ Intenso } & TIC & 3,18 & 1,1 & 6,3 & 52,3 & 40,3 \\
\hline & PCS & 3,38 & 3,3 & 4,9 & 42,9 & 48,9 \\
\hline & CA & 3,3 & 1,7 & 9,1 & 46,2 & 43 \\
\hline & EWP & 3,12 & 1,1 & 5,2 & 37,7 & 56 \\
\hline \multirow{4}{*}{$\begin{array}{l}\text { Acima da } \\
\text { média }\end{array}$} & JIT & 2,94 & 1,8 & 6,2 & 50,6 & 41,4 \\
\hline & MPT & 2,63 & 1,8 & 3,6 & 38,3 & 56,3 \\
\hline & $\mathbf{C P}$ & 2,68 & 2,8 & 9,4 & 47,5 & 40,3 \\
\hline & REN & 2,84 & 1,7 & 5,6 & 44,7 & 48 \\
\hline \multirow[b]{2}{*}{ Moderado } & $\mathbf{E S}$ & 2,49 & 4,6 & 10,1 & 45 & 40,3 \\
\hline & TER & 2,3 & 3,1 & 6,0 & 58,4 & 32,5 \\
\hline
\end{tabular}

Fonte: autores 
Uso e efetividade de práticas de gestão da produção e do trabalho: um survey da indústria brasileira

Não foi possível identificar um padrão que estabelecesse uma relação entre a extensão de uso de cada prática e o ano de sua introdução, conforme percebemos na Tabela 03. Tanto para as práticas de uso mais intenso (TEQ e GQT) quanto para aquelas de alcance ligeiramente menor (como a ES e a TER), o padrão de uso e difusão é o mesmo.

\subsection{Explicando os motivos: quais foram às razões principais para a sua introdução?}

Nas fábricas que estavam utilizando correntemente uma prática, os entrevistados foram inquiridos a identificar os principais objetivos para a introdução, distinguindo entre melhoria da qualidade, redução de custos e aumento na prontidão de resposta aos clientes. Duas outras categorias ainda pertenceram a essa classificação - "nenhuma dessas" e "duas ou mais". Elas não foram apresentadas aos entrevistados, mas foram assinaladas no caso de eles apresentarem outras respostas. Os resultados podem ser observados na Tabela 4.

Tabela 04: comparativo das razões primárias de introdução das práticas

\begin{tabular}{|c|l|c|c|l|c|}
\hline Prática & $\begin{array}{c}\text { Razão principal } \\
\text { para introdução } \\
\text { no Brasil }\end{array}$ & $\begin{array}{c}\text { Valor em \% no } \\
\text { Brasil }\end{array}$ & Prática & $\begin{array}{c}\text { Razão principal } \\
\text { para introdução } \\
\text { no Brasil }\end{array}$ & $\begin{array}{c}\text { Valor em \% no } \\
\text { Brasil }\end{array}$ \\
\hline PCS & Custo & 22,4 & CP & Qualidade & 17,2 \\
\hline ES & Responsividade & 29,2 & TER & Custo & 44,9 \\
\hline MPT & Custo & 22 & EWP & Responsividade & 32,2 \\
\hline JIT & Custo & 33,1 & CA & Qualidade & 41,9 \\
\hline TIC & Responsividade & 32,2 & REN & Custo & 17,1 \\
\hline TEQ & Qualidade & 21,3 & GQT & Qualidade & 39,2 \\
\hline
\end{tabular}

Fonte: autores

$\mathrm{Na}$ construção dessa tabela, foram considerados os objetivos isolados identificados na pesquisa, já que não há como controlar quais as respostas pensadas pelos gestores quando faziam referência a dois ou mais objetivos. Entretanto, é possível assumir que a resposta individual que mais se destaca é aquela que tem a maior possibilidade de corresponder ao objetivo primário de introdução.

\subsection{Avaliando o êxito: quais práticas são vistas como as mais e as menos bem sucedidas?}


Cada uma das práticas foi investigada quanto ao alcance das expectativas das empresas em termos de qualidade, custo e responsividade. Note-se que o gestor é chamado a refletir sobre o impacto para as três categorias de análise mesmo que uma dessas dimensões não tenha sido originariamente pensada ou referida por ele como razão primária para a introdução da prática na organização. A taxa de sucesso é uma medida obtida a partir da soma dos indicadores “muito" e "totalmente". Os resultados são apresentados na Tabela 5.

A possibilidade de sucesso de cada uma das práticas investigadas cresce à medida que as demais são utilizadas simultaneamente. Esse resultado indica claramente que o conjunto das práticas se refere a um modelo específico de organização do trabalho e da produção.

Tabela 05: Alcance de objetivos pretendidos para os fatores de qualidade, custos e responsividade

\begin{tabular}{|c|c|c|c|}
\hline \multirow{2}{*}{ Prática } & \multicolumn{3}{|c|}{ Taxa de sucesso percebido } \\
\cline { 2 - 4 } & Qualidade (\%) & Custos (\%) & Responsividade (\%) \\
\hline \multirow{2}{*}{ PCS } & 53,2 & 44,5 & 57 \\
\hline MPT & 54,8 & 47,4 & 58,5 \\
\hline JIT & 44,2 & 48,6 & 48,5 \\
\hline TIC & 42,1 & 60,2 & 60,1 \\
\hline TEQ & 69,7 & 59,4 & 67,9 \\
\hline CP & 70,9 & 52,5 & 65,9 \\
\hline TER & 67,4 & 56,4 & 41,6 \\
\hline EWP & 28,4 & 37 & 59,1 \\
\hline CA & 53,3 & 38,8 & 54,7 \\
\hline REN & 64,6 & 43 & 58,7 \\
\hline GQT & 62,1 & 53,2 & 64,4 \\
\hline & 76,5 & 51 & \\
\hline
\end{tabular}

Fonte: autores

\subsection{Projetando o futuro: quais das práticas terão uso continuado no futuro?}

Ao se posicionar sobre o grau esperado de utilização das práticas para os três anos seguintes à realização da pesquisa, o gestor faz uma dupla avaliação. Inicialmente, ele estabelece um juízo sobre a sua eficácia no momento, reforçando os posicionamentos já registrados e, em seguida, projeta para o futuro, a partir do seu repertório de informações, as potencialidades de uso continuado de cada prática. 
Uso e efetividade de práticas de gestão da produção e do trabalho: um survey da indústria brasileira

Para avaliar o potencial de uso de cada prática no futuro, foi calculada uma taxa que agrega as avaliações feitas pelos gestores quanto à sua permanência nos mesmos patamares atuais, ou se seu uso cresceria. Assim, foram somados os percentuais das categorias "o mesmo", "mais" e "muito mais". Na Tabela 06, os resultados encontram-se reunidos por agrupamentos de práticas.

Tabela 06: Expectativa de uso futuro das práticas implementadas

\begin{tabular}{|c|c|c|c|c|c|}
\hline $\begin{array}{c}\text { Práticas } \\
\text { Design e produção/ } \\
\text { Inventário e estoque }\end{array}$ & $\begin{array}{c}\text { Expectativa } \\
\mathbf{d e} \\
\text { uso futuro } \\
(\boldsymbol{\%})\end{array}$ & $\begin{array}{c}\text { Práticas } \\
\text { Organização } \\
\text { do trabalho }\end{array}$ & $\begin{array}{c}\text { Expectativa } \\
\text { de uso } \\
\text { futuro } \\
(\boldsymbol{\%})\end{array}$ & $\begin{array}{c}\text { Práticas } \\
\text { Organização } \\
\text { mais ampla da } \\
\text { produção }\end{array}$ & $\begin{array}{c}\text { Expectativa } \\
\text { de uso } \\
\text { futuro } \\
(\boldsymbol{\%})\end{array}$ \\
\hline PCS & 95,3 & TEQ & 98,2 & EWP & 96,4 \\
\hline ES & 87,7 & CP & 84,1 & CA & 97,7 \\
\hline MPT & 95 & TER & 72,2 & REN & 83,4 \\
\hline JIT & 92,9 & & & GQT & 98,7 \\
\hline TIC & 95 & & & & \\
\hline
\end{tabular}

Fonte: autores

Com a análise desses resultados, pode-se chegar a uma importante conclusão: as altas taxas identificadas para todas as práticas fortalecem a interpretação de que elas se apresentam como componentes ou partes de um modelo mais amplo que caracteriza o modo de produção na primeira década do século XX.

\section{5 À GUISA DE CONCLUSÕES: O QUE PODE SER DITO ACERCA DE CADA PRÁTICA?}

Uma síntese dos principais achados frente a cada prática de inovação investigada encontra-se no Quadro 2. Nas seções anteriores, os resultados foram dispostos de forma separada para cada questão, o que pode dificultar a percepção do perfil de adoção e da avaliação de cada prática isoladamente. Cada prática foi caracterizada em função dos resultados obtidos nas principais questões investigadas - a intensidade do seu uso, o período de introdução, os motivos que levaram à adoção, a avaliação do seu sucesso nas três dimensões (qualidade, custo e responsividade) e o seu potencial de uso no futuro quando de realização da pesquisa. Assim, o objetivo desta seção é prover um quadro referencial, resumo dos resultados apresentados. Ao se mencionar a TER, por exemplo, não se estará 
apresentando um resultado isolado, mas sua posição relativa às demais práticas sob investigação.

Para o adequado exame das informações constantes no Quadro 02, alguns esclarecimentos sobre os critérios utilizados devem ser apresentados de início:

- Com relação ao uso atual, trabalhou-se com os escores médios de uso, já que as categorias de respostas assim o permitiam. Para criar as categorias apresentadas, foram considerados os seguintes critérios: as práticas que obtiveram valores de uso localizados entre 0-1 foram classificadas como de baixo uso; aquelas que obtiveram valores situados entre 1,1 e 2,9 foram classificadas como de médio uso; e aquelas com valores de uso superiores a 3 (inclusive) foram classificadas como de alto uso.

- Com relação ao período de introdução, o critério de classificação foi o seguinte: as práticas com maiores taxas de introdução após 1997 foram classificadas como recentíssimas; as práticas introduzidas mais fortemente entre 1990 e 1997 foram classificadas como recentes; e aquelas introduzidas antes de 1989 (inclusive) classificadas como antigas.

- Quanto aos objetivos para a adoção da prática destacou-se aquele que, isoladamente, apresentou o maior contingente de respostas.

- Com relação à efetividade do uso, o critério de classificação se baseou na soma dos resultados das categorias "nada" e "pouco" para a classificação de baixo uso; "moderadamente" para a classificação de médio uso; e "muito" e "totalmente" para a classificação de alto uso.

- E, finalmente, no quesito crescimento, o critério de classificação utilizado foi: as práticas com taxa de descontinuidade de uso de até $5 \%$ foram classificadas como de alto crescimento; aquelas com taxas entre $5,1 \%$ e $15 \%$ foram classificadas como de médio crescimento; e aquelas com taxas maiores de $15 \%$ de descontinuidade de uso, classificadas como de baixo crescimento.

Veja-se, então, uma síntese dos resultados que caracterizam cada uma das práticas investigadas.

- Reengenharia de Negócios (REN) no Brasil possui uso médio, recentíssimo, e o principal objetivo para sua introdução é o de reduzir custos. Essa prática obtém um alcance acima da média para os três objetivos, embora seu potencial de crescimento seja baixo.

REAd I Porto Alegre - Edição 72 - N 2 - maio/agosto 2012 - p. 372-399 
Uso e efetividade de práticas de gestão da produção e do trabalho: um survey da indústria brasileira

- Parceria na Cadeia de Suprimentos (PCS) encontra-se entre aquelas práticas que possuem um uso mais intenso. Sua adoção também é recentíssima, tendo como principal objetivo a redução de custos. A avaliação do seu sucesso é alta, tanto para qualidade quanto para responsividade, mas não se mostra tão eficaz na redução de custos. Seu potencial de crescimento é alto.

- Terceirização (TER), apesar de ser a prática menos utilizada, encontra-se no patamar de uso médio. Sua introdução é recente (sua difusão predomina nos anos noventa, antes de 1997). É focada na redução de custos e possui um alcance baixo no que toca a esse objetivo, sendo moderadamente efetiva na melhoria da qualidade e no aumento de velocidade de resposta aos clientes. Seu potencial de uso futuro é baixo.

- Cultura de Aprendizagem (CA) é altamente utilizada e também se revela de introdução recente (forte concentração de respostas no período inicial dos anos noventa). Sua adoção busca, sobretudo, a melhoria da qualidade. Ela possui um menor sucesso na redução de custos e uma efetividade positiva em relação à qualidade. A tendência é a de ampliar o seu uso no futuro.

- Empowerment (EWP) é de introdução recentíssima e de uso alto. Essa prática possui um sucesso alto quanto à qualidade e responsividade e, a exemplo da CA, seu impacto na redução de custos é menor. Sua tendência é a de crescer ainda mais no futuro.

- Trabalho em Equipes (TEQ) é de utilização elevada e considerada uma inovação recentíssima. É mais efetiva para a melhoria da qualidade e para o aumento de velocidade de resposta aos clientes do que para a redução de custos. A tendência esperada é a de que o seu uso aumente.

- Manutenção Produtiva Total (MPT) é uma prática de uso recentíssimo, embora de média utilização. Seu foco está na redução de custos, alcançando medianamente o objetivo almejado. Sua efetividade para melhoria na qualidade é alta. Para a velocidade de resposta aos clientes, ela também obtém elevado êxito. A expectativa é que tal prática seja mais utilizada no futuro.

- Engenharia Simultânea (ES) é também uma prática recente e usada relativamente menos do que outras técnicas de gestão mais recentes. A razão primária para a sua introdução é a busca de maior responsividade. Parece alcançar adequadamente os objetivos pretendidos também em termos de responsividade, qualidade e custos. Os entrevistados não apontam para um crescimento significativo do seu uso no futuro. 
- Tecnologia Integrada Baseada em Computador (TIC) é usada relativamente mais que a maioria das outras práticas, sendo uma inovação recente, difundida, sobretudo, na primeira metade da década de 90. Demonstrou efetividade elevada para reduzir custos, aumentar a qualidade e a responsividade, sendo esse último o seu principal objetivo. A tendência para essa prática é a de expansão.

- Células de Produção $(\mathrm{CP})$ é também recente, mas de uso médio em relação à maioria das inovações. Ela se presta tanto a reduzir custos, como para melhorar a prontidão na resposta aos clientes e melhorar a qualidade. Não se mostra como uma prática que terá um crescimento significativo do seu uso no futuro.

- Just-In-Time (JIT) é uma prática considerada inovadora, recente e de uso moderado. Ela focaliza a redução de custos, sendo de grande eficácia no que se propõe. É também avaliada como produtora de elevada melhoria de qualidade e de responsividade. $\mathrm{O}$ crescimento da JIT para os próximos anos deve ser mediano.

- Gestão da Qualidade Total (GQT) é muito utilizada, sendo uma inovação recente. É bem sucedida na melhoria da qualidade, objetivo a que se propõe. Possui alto alcance dos objetivos de ampliar a velocidade de resposta e de reduzir os custos. O crescimento do uso da TQM é alto. O Quadro 2, a seguir, apresenta uma síntese desses resultados. 
Uso e efetividade de práticas de gestão da produção e do trabalho: um survey da indústria brasileira

Quadro 2: Síntese dos resultados obtidos

\begin{tabular}{|c|c|c|c|c|c|c|c|}
\hline \multirow[b]{2}{*}{ Prática } & \multirow[b]{2}{*}{ Uso } & \multirow[b]{2}{*}{ Introdução } & \multirow{2}{*}{$\begin{array}{l}\text { Principal } \\
\text { objetivo }\end{array}$} & \multicolumn{3}{|c|}{ Efetividade } & \multirow{2}{*}{$\begin{array}{l}\text { Cresci- } \\
\text { mento }\end{array}$} \\
\hline & & & & Qualidade & Custo & $\begin{array}{l}\text { Responsi- } \\
\text { vidade }\end{array}$ & \\
\hline Reengenharia & Médio & Recentíssimo & Custo & Alta & Alto & Alta & Baixo \\
\hline Parceria & Alto & Recentíssimo & Custo & Alta & Moderado & Alta & Alto \\
\hline Terceirização & Médio & Recente & Custo & Baixa & Moderado & Moderada & Baixo \\
\hline $\begin{array}{c}\text { Cultura de } \\
\text { aprendizagem }\end{array}$ & Alto & Recente & Qualidade & Alta & Moderado & Alta & Alto \\
\hline $\begin{array}{c}\text { Empowerment } \\
\text { Trabalho em }\end{array}$ & Alto & Recentíssimo & Responsividade & Alta & Moderado & Alta & Alto \\
\hline $\begin{array}{c}\text { Equipes } \\
\text { Manutenção p. }\end{array}$ & Alto & Recentíssimo & Qualidade & Alta & Alto & Alta & Alto \\
\hline $\begin{array}{c}\text { Total } \\
\text { Engenharia }\end{array}$ & Médio & Recentíssimo & Custo & Alta & Moderado & Alta & Alto \\
\hline $\begin{array}{l}\text { Simultânea } \\
\text { Tecnologia }\end{array}$ & Médio & Recente & Responsividade & Alta & Alto & Alta & Baixo \\
\hline $\begin{array}{l}\text { Integrada } \\
\text { Células de }\end{array}$ & Alto & Recente & Responsividade & Alta & Alto & Alta & Alto \\
\hline Produção & Médio & Recente & Qualidade & Alta & Alto & Alta & Baixo \\
\hline $\begin{array}{l}\text { Just in time } \\
\text { Qualidade }\end{array}$ & Médio & Recente & Custo & Alta & Alto & Alta & Médio \\
\hline Total & Alto & Recente & Qualidade & Alta & Alto & Alta & Alto \\
\hline
\end{tabular}

Fonte: autores

A partir desse quadro sintético, pode-se concluir que as práticas PCS, TEQ, TIC e GQT formam um grupo comum, bem sucedido e com possibilidades maiores de uso continuado no futuro. Além disso, são as inovações mais comuns e, embora utilizadas para uma variedade de objetivos, são relativamente bem sucedidas em comparação com as outras práticas. Um aspecto que não poderia ser negligenciado é o fato de que o uso da REN e da CP não é alto. Essas práticas ocupam nesse período as categorias das menos utilizadas, apesar de REN ser de uso moderado. Os índices de sucesso são relativamente bons em comparação com a maioria das demais práticas.

Nesse ponto, é importante destacar que, à exceção da Gestão da Qualidade Total (GQT) - uma prática que surge na década de 1980, quando já apresentava relativo grau de difusão -, as demais surgem antes desse período, embora apresentem baixa difusão de uso. A pesquisa registra respostas quanto à existência da CA, da REN, da TER, da ES e da PCS no 
período anterior à década de 60; para a TEQ, existem referências para os anos 60. Mas, como isso seria possível, se esses são movimentos relativamente recentes? São os gestores em exercício no ano de 2002 que identificam um conjunto de ações e procedimentos desenvolvidos nas suas organizações com uma determinada prática. Eles possuem um determinado conjunto de conceitos e ideias do que representa cada prática no presente, buscando, no passado, um conjunto de situações similares, que interpretam e classificam do mesmo modo. Tem-se, então, uma clara demonstração de como os gestores constroem sentidos retrospectivamente. Uma segunda inferência, que parece decorrer da anterior, é a de que cada organização implementa e desenvolve cada uma das práticas com uma característica própria e peculiar.

Os resultados, quando tomados em seu conjunto, parecem apontar na direção de patamar de eficiência para as organizações, caracterizado pela customização (flexibilidade frente à demanda), qualidade (flexibilidade da oferta) e desregulamentação (flexibilidade das relações de trabalho). A partir da compreensão de um movimento sistêmico, em que as práticas façam parte de um todo, de um padrão de organização e funcionamento da sociedade capitalista, é que se podem entender tais resultados. As respostas positivas que, de uma forma geral, se observaram frente às diversas práticas fortalecem a compreensão de que se trata de uma avaliação do sistema e do seu nível de operação.

As práticas de gestão, como materializações da divisão de trabalho, refletem essa ordem. Nesse sentido, se investigássemos outro conjunto qualquer de práticas identificadas na taxonomia proposta por Bolden et al. (1997), os resultados provavelmente seriam os mesmos, pois os princípios ordenadores do seu funcionamento são similares. A variedade das práticas pode ser entendida como um princípio normal do comportamento de exploração e adaptação das organizações ao ambiente. Residiria nesse princípio a capacidade de sobrevivência das organizações frente às mudanças incertas que se vão processando nos ambientes, cada vez mais intensamente descritos como complexos e turbulentos.

As diferenças existentes entre as empresas poderiam ser consideradas distintas formas de manifestação de um mesmo princípio administrativo, no seu ajuste a contingências específicas. As práticas mais bem sucedidas sobrevivem e prosperam, tornando-se elementos a serem copiados por outras organizações. As mal sucedidas sucumbem e perecem. 
Uso e efetividade de práticas de gestão da produção e do trabalho: um survey da indústria brasileira

\section{REFERÊNCIAS}

APPELBAUM, E.; BATT, R. The New American Workplace. Ithaca: Cornell University Press, 1994.

ARAÚJO, U.; CABRAL, S. Relações entre Inovações e Rotinas :Evidências de um Caso Empírico. REAd- Revista Eletrônica de Administração, Porto Alegre, vol. 14. n. 2, Maio/Agosto, 2008.

ARKADER, R. A Pesquisa Científica em Gerência de Operações no Brasil. RAE - Revista de Administração de Empresas, São Paulo, v. 43, nº 1, jan/mar 2003, p. 70-80.

BASTOS, A.V. B. Cognição e Ação nas Organizações. In DAVEL, E. e VERGARA, Sylvia. (org.) Gestão com Pessoas e Subjetividade. São Paulo: Editora Atlas, 2001. p.31-56.

BERTERO, C., VASCONCELOS, F. C.; BINDER, M. P. 02. RAE-Revista de Administração de Empresas, São Paulo, v.43, n.4, out/dez 2003, p.48-62.

BLOOM, N.; et al. Why do Firms in Developing Countries have Low Productivity? < Disponível em http://ssrn.com/abstract=1533430> 15 de Agosto de 2011.

BLOOM, N.; VAN REENEN, J. Measuring and Explaining Management Practices Across Firms and Countries. Discussion Paper 733. Londres: Centre for Economic Performance, London School of Economics and Political Science, 2006.

BOLDEN, R. et al. A New Taxonomy of Modern Manufacturing Practices. International Journal of Operations \& Production Management. Bradford, v. 17, $\mathrm{n}^{\mathrm{o}}$ 11, p. 1112-1130, 1997.

BOXALL, P.; PURCELL, J. Strategy and Human Resource Management. Oxford: Palgrave MacMillan. 
BRESNAHAN, T. F.; BRYNJOLFSSON, E.; HITT, L. M. Information Technology, Workplace Innovation and the Demand for Skilled Labour: Firm level Evidence. The Quarterly Journal of Economics, Oxford, vol. 117, n. 2, p.339-376, 2002.

BRYMAN, A. Social Research Methods. Oxford: Oxford University Press. 2004.

CALDAS, M. Em Busca de um Modelo Abrangente para a Difusão de Modismos Gerenciais: por que os Consultores não são os únicos a Culpar? In CALDAS, M; WOOD Jr, T. Transformação e Realidade Organizacional. São Paulo: Editora Atlas, 1999. p. 66-83.

CAPPELLI, P. e NEUMARK, D. Do "High-Performance" Work Practices Improve Establishment-Level Outcomes? Industrial and Labour Relations Review, Ithaca, vol. 54, n. 4, p. $737-775,2001$.

CLEGG, C. W. et al. An International Study of Use and Effectiveness of Modern Manufacturing Practices. Human Factors and Ergonomics in Manufacturing, Chichester, vol. 12, n.1, p. 171-191, 2002.

COELHO JÚNIOR, F. A.; BORGES-ANDRADE, J. E. Uso do Conceito de Aprendizagem em Estudos Relacionados ao Trabalho e Organizações. Paidéia, Ribeirão Preto, vol. 18, n.40, p. 221-234, 2008.

DAVIES, A. J.; KOCHHAR, A. K. A Framework for Selection of Best Practices. International Journal of Operations \& Production Management, Bradford, vol. 20, n. 10, p. 1203-1217, 2000.

DIMAGGIO, P.; POWELL, W. The Iron cage Revisited: Institutional Isomorphism and Collective Rationality. American Sociological Review, Chicago, vol. 48, n. 2, p. 147-160, 1983.

ECCLES, R., NOHRIA, N.; BERKLEY, J. Assumindo a Responsabilidade: Redescobrindo a Essência da Administração. Rio de Janeiro: Campus, 1994. 
Uso e efetividade de práticas de gestão da produção e do trabalho: um survey da indústria brasileira

EPITROPAKI, O. et al. The Use and Effectiveness of Modern Manufacturing Practices in Greece. ESRC Centre for Organization and Innovation. Institute of Work Psychology. Report, Sheffield, 2001.

GITAHY, L. A New Paradigm of Industrial Organization: the Diffusion of Technological and Managerial Innovations in the Brazilian Industry. 2000. 48 f. Dissertations ( Faculty of Social Sciences). University of Uppsala. Uppsala, 2000.

GREY, C. Organizações: O Fetiche da Mudança. RAE-Revista de Administração de Empresas, São Paulo, v.44, n.1, p. 10-25, p.10-35, jan/mar, 2004.

GODINHO, M. F.; FERNANDES, F. C. F.; LIMA, A. D. Pesquisa em Gestão da Produção na Indústria de Calçados: revisão, Classificação e Análise. Gestão e Produção, São Paulo, vol. 16, n. 2, pp. 163-186, 2009.

IDE, W; TAKAHASHI, M.; KOGI, K. The use and effectiveness of Modern Manufacturing Practices in Japan and Differences from the UK. The Journal of Science and Labour, Tóquio, vol. 75,n. 1, p. 17-29, 1999.

LESEURE, M. J. et al. Adoptions of Promising Practices: a Systematic Review of Evidence. International Journal of Management Reviews, Londres, vol. 5/6, nº 3\&4, p.169-190, 2004.

KARWOWSKI, W.; CHASE, B. System Integration in Advanced Manufacturing Technology. In HOLMAN, et al. (orgs.). The Essential of the New Workplace. Chichester: John Wiley. 2005. p.51-66.

MACHADO-SILVA, C.; FONSECA, V. Estruturação da Estrutura Organizacional: O caso de uma Empresa Familiar. RAC - Rev. Adm. Contemporânea, Curitiba, vol.14, n.esp, p.11-32, 2010.

MORAES, E.; VASCONCELOS, F. Determinantes da Heterogeneidade das Empresas Brasileiras. REAd-Revista Eletrônica de Administração, Porto Alegre, vol. 16, n. 2, p. 76101, Maio/Agosto, 2010. 
MORRISON, D. et al. The Use and Effectiveness of Modern Management Practices in Australia. University of Western Australia and University of Woolongong Report, Perth, 1999

MOTTA, P. R.. Gestão Contemporânea: A Ciência e a Arte de Ser Dirigente. Rio de Janeiro: Record, 2001.

NONAKA, I. e TAKEUCHI, H. Criação de Conhecimento na Empresa - Como as Empresas Japonesas Geram a Dinâmica da Inovação. Rio de Janeiro: Campos, 1997.

PACAGNELLA JR, A. C. et al. Influência no Desempenho Inovador no Setor de bens de Capital: Uma Análise na Indústria Paulista. REAd - Revista Eletrônica de Administração, Porto Alegre, Edição 66, vol. 16, n. 2, p. 52-75, Ma/Junho 2010.

PELISSARI, A. S.; DEFREITAS, I. V.; VANALLI, R. M. Competências Gerenciais. Um Estudo em Pequenas Empresas de Confecção. REAd-Revista Eletrônica de Administração, Porto Alegre, Edição 68, vol. 17, n. 1, p. 149-180, Jan/Abr 2011.

PRAJOGO, D.I. The Comparative Analysis of TQM Practices and Quality Performance between Manufacturing and Services Firms. International Journal of Service Industry Management, Bingley, vol.16, n.. 3, p. 217-228, 2005.

RUBERY, J. Labor Markets and Flexibility. In ACROYD, S.; THOMSON, P.; TOLBERT, P. The Oxford Handbook of Work e Organization. Oxford: Oxford University Press. 2006. p.3150.

SCHONBERGER, R. World Class Manufacturing: The lessons of Simplicity Applied. New York: Free Press. 1986.

TONELLI, M. J.et al. Produção Acadêmica em Recursos Humanos no Brasil: 1991-2000. São Paulo, RAE - Revista de Administração de Empresas, v. 43, nº 1, jan/mar 2003.

WATERSON, P.et al. The Use and Effectiveness of Modern Manufacturing Practices in the United Kingdom. Sheffield, UK: ESRC Centre for Organization and Innovation -Institute of Work Psychology, 1997. 
Uso e efetividade de práticas de gestão da produção e do trabalho: um survey da indústria brasileira

WOOD JR, T.. Fordismo, Toyotismo e Volvismo: Os caminhos da Indústria em Busca do Tempo Perdido. In (org.). Mudança Organizacional. São Paulo: Editora Atlas, 2000. p. 96-116. 